\title{
Potencialidade de fungicidas biológicos no controle de requeima do tomateiro
}

\author{
José R Souza ${ }^{1}$; Tiyoko NH Rebouças ${ }^{1}$; José MQ Luz²; Cláudio LF Amaral'; ${ }^{1}$ Roseane M Figueiredo'; \\ Ceilla MP Santana ${ }^{1}$ \\ ${ }^{1}$ UESB, C. Postal 95, 45083-900 Vitória da Conquista-BA; faelmozart@yahoo.com.br; tiyoko@uesb.edu.br; geticamaralclfuesb@ \\ bol.com.br; fraurose@hotmail.com; ceillapaiva@hotmail.com ²UFU-ICIAG, C. Postal 593, 384000-902 Uberlândia-MG; jmagno@, \\ umuarama.ufu.br
}

\section{RESUMO}

O objetivo deste trabalho foi comparar o efeito da aplicação de fungicidas com agente biológico como alternativa do controle de requeima em tomateiro híbrido Silvety. O experimento foi conduzido em campo entre 2011 e 2012 na área experimental da Universidade Estadual do Sudoeste da Bahia, Vitória da ConquistaBA. Empregou-se o delineamento em blocos casualizados com cinco tratamentos, quatro repetições e unidade experimental constituída por 12 plantas. Os tratamentos (T) foram: T1) testemunha não pulverizada; T2) Trichoderma harzianum $\left(2 \times 10^{8}\right.$ conídios $\left./ \mathrm{mL}\right)$; T3) Trichoderma longibrachiatum $\left(2 \times 10^{8}\right.$ conídios/mL); T4) metalaxyl-M + mancozeb (204 g/100L i.a/ha); T5) cloridrato de propamocarbe + fluopicolide (1000 g i.a/ha) e T6) dimetamorfe (75 g/100L i.a/ha), aplicados de forma preventiva aos 35, 42, 49 e 56 DAT (dias após o transplante). Avaliou-se a severidade da doença, a produtividade e seus componentes (número de frutos por planta, massa média dos frutos, produção). Os resultados da severidade foram utilizados para calcular a área abaixo da curva do progresso da requeima (AACPR). Para a comparação das médias, realizou-se a análise de variância e teste Tukey a $5 \%$ de probabilidade. Os fungicidas químicos promoveram maior proteção contra a requeima do tomateiro. Com cloridrato de propamocarbe + fluopicolide e metalaxyl-M + mancozeb foram obtidas as menores porcentagens de área foliar atacada pela requeima, de 2,6 e 4,36\%, respectivamente, conforme avaliação aos 65 DAT. Os isolados Trichobio01 e Trichonemate01 apresentaram eficiência protetora contra a requeima até o $58^{\circ}$ DAT. Propamocarbe + fluopicolide demonstrou eficiência no controle de requeima, apresentando a menor AACPR com 31,50 e um incremento médio de $47 \%$ na produtividade.

Palavras-chave: Solanum lycopersicum, Phytophthora infestans, Trichoderma spp., controle biológico.

\begin{abstract}
Potential of biologic fungicide in controlling late blight of tomato

This study aimed to compare the efficiency of chemicals and biological agents in the alternative control of $P$. infestans in tomato hybrid Silvety. The experiment was carried out in the experimental area of the State University of Southwest Bahia, Brazil in a randomized block design with five treatments and four replications. The experimental unit consisted of 12 plants. The treatments (T) were: T1) nonsprayed check; T2) Trichoderma harzianum; T3) Trichoderma longibrachiatum; T4) metalaxyl-M + mancozeb; T5) propamocarb hydrochloride + fluopicolide, and T6) dimetamorph. Besides the evaluation of disease severity, the following variables were evaluated: number of fruits per plant, mean fruit weight and yield. The severity assessments were used to calculate area under the disease curve of the late blight progress (AACPR). The results were submitted to analysis of variance and to the Tukey test (5\%). The chemical fungicides, regardless of their mode of action, gave a greater protection against late blight. Hydrochloride propamocarbe + fluopicolide and metalaxyl-M + mancozeb resulted in the lowest rates of disease foliar area, 2.6 and $4.36 \%$, respectively, 65 days after transplant (DAT). The biological control agents T. harzianum and $T$. longibrachiatum showed protective efficiency against the late blight until 58 DAT. Propamocarbe + fluopicolide had the lowest value of the AACPR (31.50) and an average increase of $47 \%$ in productivity in relation to the nonsprayed treatment.
\end{abstract}

Keywords: Solan8um lycopersicum, Phytophthora infestans, Trichoderma spp., biological control.

(Recebido para publicação em 20 de maio de 2013; aceito em 31 de janeiro de 2014)

(Received on May 20, 2013; accepted on January 31, 2014)

$\mathrm{O}^{\prime \prime}$ tomateiro (Solanum lycopersicum) é uma das mais expressivas culturas no cenário agrícola mundial, constituindo importante produto para o comércio in natura e de processados.

A tomaticultura encontra-se disseminada em todo território nacional, sendo as regiões Sudeste e Centro-Oeste os principais centros de produção para os tomates de mesa e indústria, respectivamente. De acordo com dados do IBGE (2012), a produção nacional atingiu 4,42 milhões de toneladas na safra de 2012. O maior produtor de tomate do país é o estado de Goiás, com cerca de 1,14 milhão de toneladas, representando cerca de $31,3 \%$ da produção nacional. O Nordeste, em 2012, teve uma participa- ção de 11,1\% e a Bahia uma participação de $4,9 \%$ (IBGE, 2012).

O tomate é difícil de ser produzido pelo fato de ser sujeito a muitas doenças (Lopes \& Ávila, 2005) e, para obter resultados satisfatórios exige cuidados especiais (Camargo et al., 2006).

A requeima, causada pelo oomiceto Phytophthora infestans, caracteriza- 
-se por ser uma doença agressiva e de grande poder destrutivo, afetando severamente folhas, hastes, frutos e pecíolo, podendo limitar ou até mesmo impedir o cultivo econômico do tomateiro sob condições de alta umidade e baixas temperaturas (Lopes \& Ávila, 2005). Apesar de ser uma doença típica de épocas frias e úmidas, epidemias importantes da doença podem ser verificadas no Brasil Central, devido ao abaixamento brusco de temperatura, favorecido pelas chuvas constantes (Mizubuti, 2001). A região sudoeste da Bahia, sobretudo Vitória da Conquista-BA, está localizada próxima a Chapada Diamantina-BA, importante região na produção de tomate de mesa e, entretanto, de clima favorável ao desenvolvimento da requeima.

A importância das doenças causadas por $P$. infestans fez com que o emprego de fungicidas químicos se tornasse a principal forma de controle desse patógeno. O uso de fungicidas para o controle da requeima tem sido recomendado dentro de programas multidisciplinares de manejo, e com o advento dos fungicidas anti-oomicetos, progressos significativos foram obtidos no controle da requeima (Töfoli et al., 2000). Estes avanços foram possíveis em função das novas características apresentadas por estes produtos, tais como elevada fungitoxicidade, ação protetora e curativa, sistemicidade e boa persistência nos tecidos (Uesugi, 1998).

Por outro lado, os agrotóxicos nem sempre apresentam resultados satisfatórios e duradouros; o uso de grandes quantidades de agrotóxicos na cultura, sendo a maioria prejudicial aos ecossistemas, bem como seus resíduos em frutos, são sérias ameaças à saúde humana (Barreto \& Scaloppi, 2000). Na busca por métodos de menor impacto ambiental e à saúde humana, o controle biológico é uma das alternativas promissoras, por apresentar especificidade ao alvo, utilizar diferentes meios para atingi-lo, restringindo as chances de selecionar linhagens resistentes (Saito et al., 2009).

Espécies de Trichoderma spp. desempenham um papel importante no controle de patógenos de plantas, especialmente do solo (Tran, 2010). A aplicação de Trichoderma no solo em casa de vegetação ou no campo reduziu a severidade de doenças, como também aumentou o crescimento da planta (Harmann, 2000). Estudos com isolados de Trichoderma hazianum foram eficazes na redução de incidência de Botrytis cinerea (Lisboa et al., 2007).

Embora o uso de Trichoderma spp. como antagonista a fitopatógenos tenha se tornado uma constante, estudos dos seus efeitos no controle de $P$. infestans em tomateiro em campo, ainda são escassos. Diante do exposto, o presente trabalho teve como objetivo avaliar o efeito de fungicidas e espécies de Trichoderma spp. como alternativa no controle de Phytophthora infestans em tomateiro híbrido Silvety.

\section{MATERIAL E MÉTODOS}

O experimento foi conduzido na área experimental da Universidade Estadual do Sudoeste da Bahia, Vitória da Conquista-BA (14'53'51's, 4048'23'O, altitude média $900 \mathrm{~m}$ ). A precipitação média é de 700 a 1200 mm/ano. O solo da área experimental foi classificado como Cambissolo Háplico Tb, Distrófico, com textura média, em topografia suavemente ondulada e boa drenagem. Foi utilizado o tomateiro híbrido Silvety da empresa Syngenta. O preparo do solo foi feito convencionalmente com arado, e sulcado em seguida, como recomendado para a cultura do tomateiro (Macedo et al., 2005).

$\mathrm{O}$ delineamento experimental utilizado foi em blocos casualizados constituído por seis tratamentos: [T1) testemunha; T2) Trichoderma harzianum isolado Trichobio $01\left(2 \times 10^{8}\right.$ conídios/ mL); T3) Trichoderma longibrachiatum isolado Trichonemate $01\left(2 \times 10^{8}\right.$ coní$\operatorname{dios} / \mathrm{mL})$; T4) metalaxyl-M + mancozeb (204g/100L i.a/ha), T5) cloridrato de propamocarbe + fluopicolide $(1000 \mathrm{~g}$ i.a/ha) e T6) dimetamorfe (75 g/100 L i.a/ha] e quatro repetições. Os isolados utilizados no experimento pertencem à coleção de fungos de antagonista do Laboratório da empresa BIOFUNGI. Cada parcela experimental foi composta por 12 plantas úteis, distribuídas em duas fileiras com seis plantas em um espaçamento de 1,2 m entre linhas e 0,6 m entre plantas.

A semeadura foi realizada no dia 07/01/2012, de forma manual, em bandejas de polietileno contendo 200 células, e utilizou-se o substrato à base de vermiculita. Após a semeadura e irrigação, as bandejas foram acondicionadas em estufa, na qual permaneceram por 35 dias. Aos 35 dias, procedeu-se o transplante, com as mudas medindo aproximadamente $8 \mathrm{~cm}$ de altura e com quatro folhas definitivas. As mudas referentes aos tratamentos T2 (Trichoderma harzianum isolado Trichobio 01) e T3 (Trichoderma longibrachiatum isolado Trichonemate 01) foram imersas em uma solução contendo água e esporos ( $2 \times 10^{8}$ conídios $\left./ \mathrm{mL}\right)$ dos respectivos fungos, durante 15 minutos e, em segui$\mathrm{da}$, levados a campo. Os tratos culturais adotados foram a capação e a desbrota. O controle fitossanitário foi realizado mediante a necessidade, quando da ocorrência de pragas ou doenças, e de forma igual para todos os tratamentos. Adotou-se o sistema de irrigação por aspersão.

A aplicação dos fungicidas químicos e agentes biológicos testados no experimento foi efetuada de forma preventiva aos 35, 42, 49 e 56 DAT (dias após o transplante). Para aplicação dos tratamentos, empregou-se pulverizador costal manual de 20 L com bico cônico, calibrado para a aplicação de um volume de calda de 1000 L/ha.

Para avaliar a severidade da requeima nas plantas utilizou-se a escala diagramática de James (1971), com valores de $1,10,25$ e $50 \%$ em intervalos de sete dias e cinco avaliações aos 37, 44, 51, 58 e 65 DAT respectivamente.

Os valores médios de severidade de cada tratamento foram utilizados para calcular as curvas de progresso da requeima. A partir das curvas de progresso, foram calculados os valores da área abaixo da curva de progresso da requeima (AACPR) para cada tratamento, conforme metodologia proposta por Shaner \& Finney (1977).

Colheram-se os frutos das parcelas úteis de forma manual, conforme atingissem a maturação fisiológica. Após a colheita, foram avaliados o número de fruto por planta (contagem total dos 
frutos e divisão pelo número de plantas da respectiva parcela); peso médio $(\mathrm{g})$ dos frutos (divisão da produção total dos frutos pelo número total de frutos da parcela); produção por planta (peso total dos frutos da parcela dividido pelo número de plantas úteis); produtividade ( $\mathrm{t} / \mathrm{ha}$ ) (pesagem dos frutos das plantas úteis e cálculo da média por planta e o equivalente por hectare).

A análise estatística foi realizada utilizando-se o programa STATISTIX, versão 9.0 (Tallahassee, 2008), procedendo-se a análise de variância. Posteriormente, as médias dos tratamentos foram separadas pelo teste de Tukey, a $5 \%$ de probabilidade. Os dados da AACPR foram previamente transformados em raiz quadrada de $\mathrm{x}+0,5$.

\section{RESULTADO E DISSCUSSÃO}

A ocorrência natural da requeima e as condições favoráveis de umidade e temperatura ao desenvolvimento da doença, aliada à suscetibilidade do híbrido Silvety, permitiram elevados níveis de requeima nas testemunhas, o que favoreceu a diferenciação dos tratamentos.

O tratamento T1 (testemunha não pulverizada) apresentou severidade final de 38,8\%. Nos tratamentos T2 ( $T$. harzianum isolado Trichobio 01) e T3 (T. longibrachiatum isolado Trichonemate 01 ), os valores de severidade final foram de 11,3 e 11,2\% respectivamente. Os valores de severidade nos tratamen- tos T4 (metalaxyl-M + mancozeb), T5 (cloridrato de propamocarbe) e T6 (dimetamorfe) foram de 4,36, 2,6 e 7,5\% respectivamente (Figura 1).

O tratamento testemunha diferenciou-se em relação aos demais a partir dos 51 DAT e permaneceu significativa até a avaliação final aos 65 DAT. As menores porcentagens de área foliar afetada pela doença foram verificadas nas plantas tratadas com cloridrato de propamocarbe + fluopicolide e metalaxyl-M + mancozeb aos 65 DAT, seguido de dimetamorfe (Figura 1).

Os antagonistas T. harzianum e $T$. longibrachiatum apresentaram ação protetora contra a requeima até 58 DAT e superior à testemunha em todas as avaliações. A eficácia de controle das espécies de Trichoderma avaliadas pode estar associada a suas características intrínsecas como a habilidade de sobrepujar os mecanismos de defesa dos patógenos, competir com microrganismo do solo e resistir aos fatores abióticos (Bae \& Knudsen, 2005).

Os fungicidas químicos apresentaram eficiência até 65 DAT. Os tratamentos apresentaram eficácia diferenciada em função do seu respectivo modo de ação. Os melhores níveis finais de controle foram obtidos com fungicidas químicos em detrimento dos produtos biológicos. Tal superioridade pode ser justificada pelo fato dos fungicidas químicos testados apresentarem características positivas com ação imunizante e boa persistência nos tecidos (Schwinn \& Staub, 1995).
Apesar da menor severidade nos tratamentos T2 (T. harzianum) e T3 ( $T$. longibraciatum), o nível de doença no campo foi considerado alto, quando comparado com a testemunha. A redução da severidade nos tratamentos pode ser atribuída à produção de antibiótico e enzimas de degradação da parede celular dos oomicetos (Sharma et al., 2009). A ação micoparasíticas observada neste estudo sugere que Trichoderma spp. possui um bom potencial no controle de $P$. infestans em condições de campo, embora seja necessário maior número de pesquisas, no que diz respeito às concentrações de conídios, doses e épocas de aplicações. Ações micoparasíticas similares de cepa de Trichoderma spp. foram relatadas in vitro contra patógenos relacionados, $P$. cinnamomi (Pugeg \& Ian, 2006), P. capsici (Ezziyyani et $a l ., 2007)$ e em condições de casa de vegetação, $P$. palmivora (Mpika et al., 2009), P. infestans (Zegeye et al., 2011).

$\mathrm{O}$ alto potencial de controle da requeima apresentado por cloridrato de propamocarbe + fluopicolide, e metalaxyl-M + mancozeb, neste trabalho, estão de acordo com as observações de Töfoli (2012) e Rodrigues et al. (2000). Estudos realizado por Reiter et al. (1995) demonstram que o propamocarbe caracteriza-se por apresentar importante ação preventiva e curativa contra a requeima, tanto em tomate como em batata, reduzindo a produção de esporângios entre 70 e 100\%.

As plantas pulverizadas com Trichoderma spp. reduziram a

Tabela 1. Número de frutos por planta, massa fresca de frutos (g), produção (kg/planta) e produtividade (kg/ha) de tomateiro híbrido Silvety tratado ou não com produtos químicos e biológicos no controle da requeima (Phytophthora infestans) [number of fruits per plant, mean fruit weight $(\mathrm{g})$, production $(\mathrm{kg} / \mathrm{plant})$ and productivity $(\mathrm{kg} / \mathrm{ha})$ of tomato hybrid Silvety treated or not with chemical and biological control for late blight]. Vitória da Conquista, UESB, 2013.

\begin{tabular}{lcccc}
\hline Tratamentos & $\begin{array}{c}\mathbf{N}^{\mathbf{0}} \text { frutos/plan- } \\
\text { ta }\end{array}$ & $\begin{array}{c}\text { Massa fresca de } \\
\text { frutos (g) }\end{array}$ & $\begin{array}{c}\text { Produção (kg/ } \\
\text { planta) }\end{array}$ & $\begin{array}{c}\text { Produtividade } \\
\text { (t/ha) }\end{array}$ \\
\hline testemunha não tratada & $4,45 \mathrm{c}$ & $194,54 \mathrm{~b}$ & $2,79 \mathrm{c}$ & $76,27 \mathrm{c}$ \\
T. harzianum & $6,86 \mathrm{ab}$ & $209,61 \mathrm{ab}$ & $8,21 \mathrm{ab}$ & $122,34 \mathrm{ab}$ \\
T. longibrachiatum & $6,21 \mathrm{~b}$ & $216,16 \mathrm{ab}$ & $7,76 \mathrm{bc}$ & $115,02 \mathrm{bc}$ \\
metalaxyl-M + mancozeb & $7,33 \mathrm{ab}$ & $210,96 \mathrm{ab}$ & $9,04 \mathrm{ab}$ & $133,28 \mathrm{ab}$ \\
cloridrato de propomocabe + fluopicolide & $7,89 \mathrm{a}$ & $238,45 \mathrm{a}$ & $10,80 \mathrm{a}$ & $160,67 \mathrm{a}$ \\
dimetamorfe & $7,4 \mathrm{ab}$ & $218,15 \mathrm{ab}$ & $9,42 \mathrm{ab}$ & $138,43 \mathrm{ab}$ \\
\hline CV (\%) & 10,32 & 9,41 & 17,94 & 17,84 \\
\hline
\end{tabular}

Médias seguidas da mesma letra na coluna não diferem entre si pelo teste de Tukey ao nível de 5\% de probabilidade (means followed by the same letter in the column do not differ by Tukey test at $5 \%$ probability). 


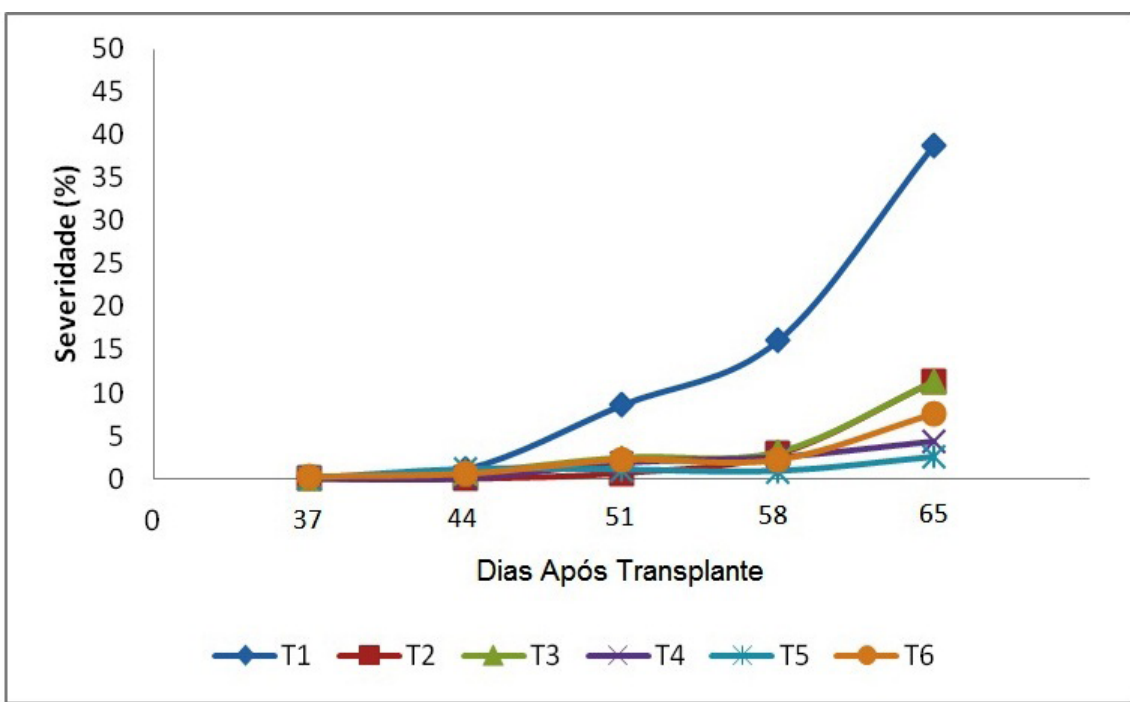

Figura 1. Curva de progresso da requeima para os tratamentos T1 (testemunha); T2 (Trichoderma harzianum); T3 (Trichoderma longibrachiatum); T4 (metalaxyl-M + mancozeb); T5 (cloridrato de propamocarbe + fluopicolide); T6 (dimetamorfe) [late blight progress curve for the treatments T1 (unsprayed check); T2 (Trichoderma harzianum); T3 (Trichoderma longibrachiatum); T4 (metalaxyl-M + mancozeb); T5 (propamocarb hydrochloride + fluopicolide); T6 (dimetamorph)]. Vitória da Conquista, UESB, 2013.

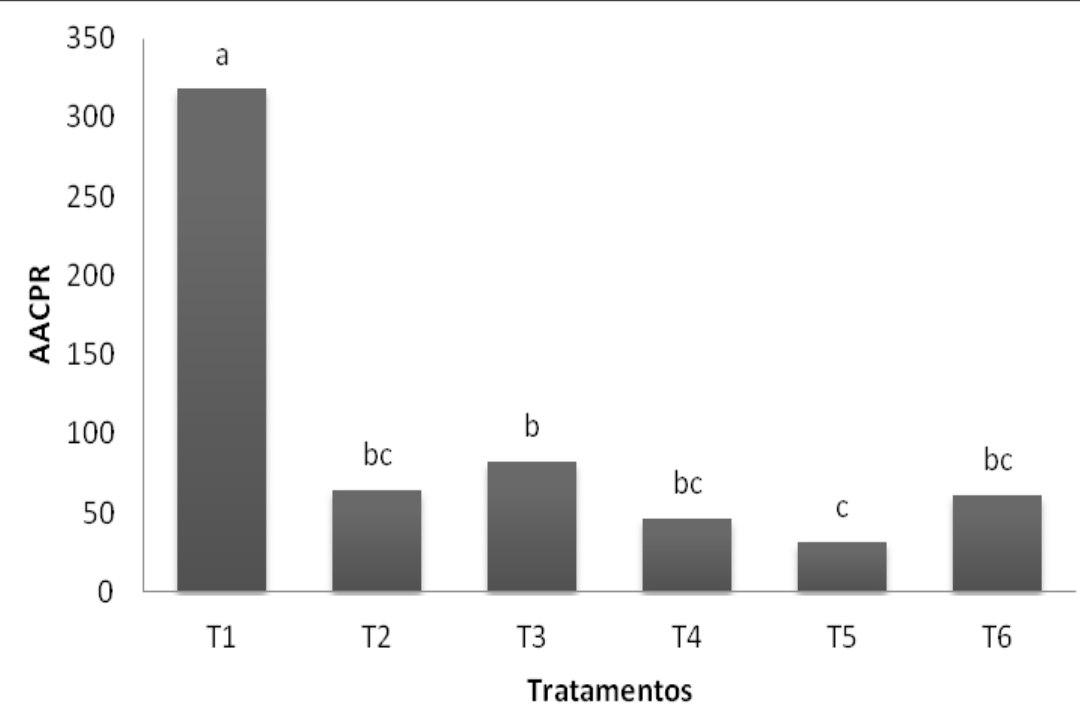

Figura 2. Área abaixo da curva de progresso da requeima em tomateiro híbrido Silvety. $\mathrm{T} 1=$ testemunha; $\mathrm{T} 2=$ Trichoderma harzianum $; \mathrm{T} 3=$ Trichoderma longibrachiatum $; \mathrm{T} 4=$ metalaxyl-M + mancozeb; T5= cloridrato de propamocarbe + fluopicolide; $\mathrm{T} 6=$ dimetamorfe (area under the curve of progress of late blight on tomato hybrid Silvety. $\mathrm{T} 1=$ control; $\mathrm{T} 2=$ Trichoderma harzianum; $\mathrm{T} 3=$ Trichoderma longibrachiatum; $\mathrm{T} 4=$ metalaxyl-M + mancozeb; $\mathrm{T} 5=$ propamocarb hydrochloride + fluopicolide; $\mathrm{T} 6=$ dimetamorph). Vitória da Conquista, UESB, 2013.

severidade da doença em comparação com o controle que obteve o maior índice entre os tratamentos, atingindo 317,9 .

Os menores valores de AACPR (menores gravidade da doença) foram registrados em cloridrato de propamocarbe $(31,50)$, seguindo por metalaxyl-M + mancozeb $(43,03)$, dimetamorfe $(61,44)$, T. harzianum
$(64,34)$, e $T$. longibrachiatum $(82,08)$ (Figura 2).

Os resultados obtidos a partir da aplicação do agente biológico em campo reduziram significativamente os efeitos da fase de infecção foliar da requeima. É possível que mais de um mecanismo antagônico esteja envolvido na redução da doença.

Estudos realizados por Töfoli et al. (2012) no controle da requeima mostraram que os maiores níveis de ação residual na planta foram obtidos com produtos móveis, sendo as primeiras reduções de controle observadas a partir dos 12 DAT.

Não houve diferenças significativas entre os tratamentos em relação à quantidade inicial da doença.

A elevada ação protetora de cloridrato de propamocarbe + fluopicolide, obtida neste estudo, pode ser explicada pela ação translaminar de fluopicolide e sistêmica de propamocarbe.

A eficiência dos antagonistas, durante a condução do experimento, pode estar relacionada a diferentes mecanismos de ação (antibiose, hiperparasitismo ou competição) e a variabilidade desse mecanismo pode ser compreendida entre as espécies, tamanho da área de ação, bem como a interação hospedeiro versus parasita (Krugner \& Bacchi, 1995).

Quanto ao número de frutos por planta e produtividade, todos os tratamentos foram superiores à testemunha. Cloridrato de propamocarbe + fluopicolide proporcionou os melhores resultados, sendo superior aos demais tratamentos (Tabela 1).

Os maiores incrementos de massa fresca e produção de frutos foram verificados nas parcelas tratadas com cloridrato de propamocarbe + fluopicolide. Todos os tratamentos proporcionaram aumento significativo da produção e produtividade em relação à testemunha (Tabela 1).

A menor produtividade do tratamento testemunha está relacionada com uma maior incidência de requeima, assim, uma maior área foliar foi necrosada pela doença e, consequentemente, menor área fotossinteticamente ativa. $\mathrm{O}$ uso de fungicidas em epidemias severas de requeima pode contribuir de forma direta 
sobre o valor comercial da produção. A aplicação de Trichoderma spp. para o controle da requeima deve ser considerado como parte integrante de programa de controle integrado, em vez da utilização de um método isoladamente, tendo em vista o seu potencial micoparasítico.

\section{AGRADECIMENTOS}

Ao Programa de Pós Graduação em Agronomia da Universidade Estadual do Sudoeste da Bahia (UESB).

\section{REFERÊNCIAS}

BAE YS; KNUDSEN GR. 2005. Soil microbial biomass influence on growth and biocontrol efficacy of Trichoderma harzianum. Biological Control 32: 236-242.

BARRETO M, SCALOPPI EG. 2000. Sistemas de previsão de hortaliças. In: Zambolim L (ed) Manejo integrado de doenças, pragas e plantas daninhas. Viçosa: Suprema Gráfica e Editora. p. 169-186.

CAMARGO FP; ALVES HSA; CAMARGO FILHO WP; VILELA NJ. 2006. Cadeia produtiva de tomate industrial no Brasil: resenha da década de 1990, produção regional e perspectivas. Informações Econômicas 36: 7-20.

EZZIYYANI M; REQUENA ME; EGEAGILABERT C; CANDELA ME. 2007. Biological control of Phytophthora root of pepper using Trichoderma harzianum and Streptomyces rochei in combination. Journal of Phytopathology 155: 324-349.

HARMAN G. 2000. Myths and dogmas of control. Changes in perceptions derived from research on Trichoderma harzianum T-22. Plant Disease 84: 393.

INSTITUTO BRASILEIRO DE GEOGRAFIA E ESTATÍSTIVA (IBGE). 2012. Levantamento sistemático da produção agrícola. Disponível em: http://www.ibge.gov.br/home/estatistica/ indicadores/agropecuaria/lspa/lspa_201212. pdf. Acessado em 14 de janeiro de 2013.

JAMES WC. 1971. An illustrated series of assessment keys for plants diseases. Their preparation and usage. Cannadian Plant Diseas Survey p.39-65.

KRUGNER TL; BACCHI LMA. 1995. Fungos, In: BERGAMIM FILHO A; KIMATI H; AMORIN L. Manual de Fitopatologia 3 ed. São Paulo: Agronômica Ceres. p.46-95.

LISBOA BB; BOCHESE CC; VARGAS LK; SILVEIRA JRP; RADIN B; OLIVEIRAAMR. 2007. Eficiência de Trichoderma harzianum e Gliocladium viride na redução da incidência de Brotytis cinera em tomateiro cultivado sob ambiente protegido.

LOPES CA; ÁVILA AC. 2005. Doenças do tomateiro. Brasília: EMBRAPA/CNPH. 152p.

MACEDO JR; CAPACHE CL; MELO AS; BHERING SB. 2005. Recomendações técnicas para a produção de tomate ecologicamente cultivado. Brasília: EMBRAPA. 10p.

MIZUBUTI ESG. 2001. Requeima ou mela da batata e do tomate. In: LUZ EDNSAF: MATSUAKA K; BEZERRA JL (ed) Doenças causadas por Phytophthora no Brasil. Campinas: Livraria Editora Rural. p. 100-174.

MPIKA J; KÉBÉ IB; ISSALI AE; N'GUESSA FK; PRUZHININA S; ZELAZOWSKA MK; KUBICEK CP; AKE S. 2009. Antagonist potential of Trichoderma indignus isolats for biological control of Phytophthora palmivora the causative agent of black pod disease on cocoa (Theobroma cacao L.) in Côte d'Lvoire. African Journal of Biotechnology 8: 5280-5293.

PUGEG INA; IAN DG. 2006. Mycoparasitic and antagonistic inhibition on Phytophthora cinnamon rands by microbial agents isolated from manure composts. Plant Pathology 5: 291-298.

REITER B; WENZ M; BUSCCHHAUS H; BUCHENAUER H. 1995. Effect of propamocarb hydrochloride on Phytophthora infestans in vitro and in potato and tomato plant. Gesunde Pflanzen 47: 43-50.

RODRIGUES C; RIBEIRO LG; LOPES JC; FREITAS FS; AZEVEDO LAS. 2000.
Eficiência do metalaxyl no controle da requeima do tomateiro. Horticultura Brasileira 8: 65-67.

SAITO LR; SALES LLSR; MARTINEKOSKI L; REFFATTI TO. 2009. Aspects of the effect fungu Trichoderma spp. in biocontrol of pathogens of agricultural crops. 2: 209-216.

SCHWINN F; STAUB T. 1995. Oomycetes fungicides. In: LUYR H(ed). Modern selective fungicides. Jena: Gustav Fischer. p.323-346.

SHANER G; FINNEY RE. 1977. The effect of nitrogen fertilization on the expression of slow mildewing resistance in Knox wheat. Phytopathology 67: 1051-1056.

SHARMA K; KUMAR M; MISRA RM. 2009. Morphological, biochemical and molecular characterization of Trichoderma harzianum isolates for their efficacy as biocontrol agents. Journal of Phytopathology 157: 51-56.

TALLAHASSEE. 2008. STATISTIX, Statistix 9.0. Analytical software. USA.

TÖFOLI JG; DOMINGUES RJ; GARCIA JO. 2000. Desempenho de fungicidas no controle da requeima do tomateiro. Horticultura Brasileira 18: 350-351.

TÖFOLI JG; MELO PCT; GARCIA JRO. 2012. Ação protetora, residual, curativa e anti esporulante de fungicidas no controle da requeima e da pinta preta da batata em condições controladas. Arquivo Instituto Biológico 79: 209-221.

TRAN H; FICKE A; ASIIMWE T; HOFTE M; RAAIJMAKERS JM. 2010. Role of cyclic lipopeptide massetolide A in biological control of Phytophthora infestans and in colonization of tomato plants by Pseudomonas fluorescens. New Phytologist 175: 731-742.

UESUGI Y. 1998. Fungicides classes. Chemistry, uses and mode of action. In: HUTSON D; MYAMOMOTO J. (eds). Fungicide activity. Hamburg: John Wiley Sons. p.23-53.

ZEGEYE ED; SANTHANAN A; IGORFU D; TESSERA M; KASSA B. 2011. Biocontrol activity of Trichoderma viride and Pseudomonas fluorescens against Phytophthora infestans under greenhouse conditions. Journal of Agriculture Techology 7: 1589-1602. 